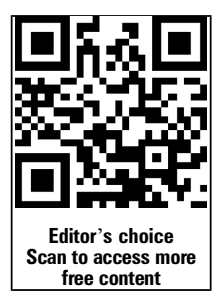

free content
Department of Ophthalmology, University of Kiel, University Medical Center SchleswigHolstein, Kiel, Germany

\section{Correspondence to} Professor Alexa Klettner Department of Ophthalmology, University of Kiel, University Medical Center SchleswigHolstein, Arnold-Heller Str. 3, Haus 25, Kiel D-24105, Germany;

aklettner@auge.uni-kiel.de

Received 26 November 2014 Revised 6 January 2015 Accepted 9 February 2015 Published Online First 4 March 2015

\section{CrossMark}

To cite: Klettner $A$, Grotelüschen S, Treumer $\mathrm{F}$, et al. Br J Ophthalmol 2015;99:864-869.

\title{
Compatibility of recombinant tissue plasminogen activator (rtPA) and aflibercept or ranibizumab coapplied for neovascular age-related macular degeneration with submacular haemorrhage
}

\author{
Alexa Klettner, Simon Grotelüschen, Felix Treumer, Johann Roider, Jost Hillenkamp
}

\section{ABSTRACT}

Background/aims Subretinal coapplication of recombinant tissue plasminogen activator (rtPA) and vascular endothelial growth factor (VEGF)-antagonists is a new treatment option for age-related macular degeneration complicated by submacular haemorrhage. Here, we investigate the compatibility of rtPA and aflibercept or ranibizumab in vitro because intraoperatively, rtPA or rtPAinduced plasmin may cleave aflibercept or ranibizumab.

Methods Aflibercept and ranibizumab, respectively, were incubated with rtPA or plasmin, separated in gel electrophoresis and stained with Coomassie or silver. The antiangiogenic activity of the VEGF-antagonists was quantified by VEGF-ELISA after incubation with the supernatant of primary porcine retinal pigment epithelium cell cultures.

Results In electrophoresis, ranibizumab displayed no additional fragments when it was coapplied with rtPA or plasmin. Its VEGF-inhibiting efficacy remained unchanged in coapplication with rtPA with or without blood, or plasmin. rtPA did not cleave or functionally compromise aflibercept. When aflibercept was coapplied with plasmin, electrophoresis displayed additional bands in Coomassie (30 kDa, $27 \mathrm{kDa}, 19 \mathrm{kDa}, 15 \mathrm{kDa}$ ) and silver staining (31 kDa, $26 \mathrm{kDa}, 21 \mathrm{kDa}, 19 \mathrm{kDa}, 15 \mathrm{kDa}$ ). While at a clinical dosage $(800 \mu \mathrm{g} / \mathrm{mL})$ VEGF was inhibited by aflibercept when coapplied with plasmin, at borderline concentrations (400 ng/mL) VEGF-binding ability of aflibercept was abolished.

Conclusions Ranibizumab is not cleaved or functionally compromised by rtPA or plasmin. Aflibercept is cleaved and its VEGF-binding ability is reduced when coapplied with plasmin. In clinical practice, rtPA and ranibizumab can be coapplied as a treatment for neovascular age-related macular degeneration with submacular haemorrhage while the antiangiogenic activity of aflibercept may be compromised when coapplied with rtPA in the presence of plasmin.

\section{INTRODUCTION}

Without treatment, the prognosis of neovascular age-related macular degeneration (AMD) complicated by submacular haemorrhage $(\mathrm{SMH})$ is usually poor because the underlying choroidal neovascularisation (CNV) lesion progresses and the resolution of the haemorrhage is associated with the formation of a macular scar. ${ }^{1}$ In addition, damage of retinal tissue by SMH has been attributed to a reduction nutrient supply, ${ }^{2}$ shrinkage of outer retinal layers and release of toxic substances. ${ }^{3-5}$
In the past, visual acuity often improved after successful displacement of SMH by recombinant tissue plasminogen activator (rtPA) and gas but then frequently deteriorated because of progression of the underlying CNV. Since the advent of antivascular endothelial growth factor (anti-VEGF) pharmacotherapy, new aspects have been added to the management of neovascular AMD with SMH. Anti-VEGF agents delivered by intravitreal injection are a safe and effective treatment for neovascular AMD for up to 2 years. ${ }^{6} 7$ In view of these clinical results, a combined intraocular application of rtPA and anti-VEGF agents could represent a significant improvement of current treatment concepts for AMD with SMH..$^{8-11}$

While the application of rtPA can help to prevent a toxic effect of SMH by effective displacement from the fovea, simultaneous application of anti-VEGF agents could potentially prevent $\mathrm{CNV}$ progression. ${ }^{8-14}$ We have previously reported promising results of a clinical case series of pars plana vitrectomy with subretinal coapplication of rtPA and bevacizumab followed by intravitreal fluid-gas exchange. ${ }^{8} 9$ However, clinicians may choose the approved drugs ranibizumab or aflibercept rather than off-label bevacizumab. In addition, in our case series $20 \%$ of eyes with SMH treated with rtPA and bevacizumab bled again. ${ }^{15}$ In such a recurrent $\mathrm{SMH}$ following initial treatment with rtPA and bevacizumab, clinicians may decide to repeat treatment but to change the anti-VEGF agent. While we have previously shown the compatibility of rtPA and bevacizumab in vitro, ${ }^{15}$ it is unclear whether rtPA and ranibizumab or aflibercept are also compatible.

rtPA is a serine protease, cleaving plasminogen into active plasmin which in turn degrades the fibrin matrix constituting blood clots. In addition, other proteins such as hepatocyte growth factor or platelet derived growth factor are also cleaved by rtPA. ${ }^{16}{ }^{17}$ Hence, it is uncertain whether in coapplication either rtPA itself, or rtPA-generated plasmin, or other undetermined proteases activated during fibrinolysis of a subretinal blood clot may cleave and thereby functionally inactivate ranibizumab or aflibercept.

The aim of this study was therefore to investigate the compatibility of rtPA and aflibercept or ranibizumab in an established in vitro model of intraoperative coapplication, assessing potential cleavage as well as functionality. 


\section{MATERIAL AND METHODS}

Cell culture

Retinal pigment epithelium (RPE) cell culture was conducted as previously described. ${ }^{18}$ In order to collect supernatant for ELISA, supernatant was collected after $4 \mathrm{~h}$. The supernatant was centrifuged $\left(13000 \mathrm{rpm}, 4^{\circ} \mathrm{C}, 5 \mathrm{~min}\right)$ and kept at $-20^{\circ}$ until further use.

\section{Interaction studies}

The coapplication of rtPA and aflibercept, rtPA and ranibizumab, plasmin and aflibercept, and plasmin and ranibizumab and, in addition, plasmin and bevacizumab, was tested. In all experiments, aflibercept (Eylea, Bayer, Leverkusen, Germany, $40 \mathrm{mg} / \mathrm{mL}$ ), ranibizumab (Lucentis, Novartis, Basel, Switzerland, $10 \mathrm{mg} / \mathrm{mL}$ ) and rtPA (Actilyse, Boehringer Ingelheim, Germany $0.2 \mathrm{mg} / \mathrm{mL}$ ), were used. Also, bevacizumab (Avastin, Roche Germany, $25 \mathrm{mg} / \mathrm{mL}$ ) was used.

\section{Recombinant tissue plasminogen activator}

To simulate intraoperative conditions, ${ }^{8} 9$ equal volumes of the respective drug and rtPA were used to ensure their usual relative concentrations. We incubated $5 \mu \mathrm{L}(200 \mu \mathrm{g})$ aflibercept as well as $5 \mu \mathrm{L}(50 \mu \mathrm{g})$ ranibizumab, respectively, with $5 \mu \mathrm{L} \mathrm{rtPA}(1 \mu \mathrm{g})$ for $2 \mathrm{~h}$ at $37^{\circ} \mathrm{C}$ to a final volume of $10 \mu \mathrm{L}$. As controls, aflibercept or ranibizumab were incubated without rtPA, and rtPA was incubated on its own for the same period of time. The mixtures were collected and separated in sodium dodecyl sulfate polyacrylamide gel electrophoresis (SDS-PAGE).

\section{Plasmin}

The interaction of plasmin with the VEGF inhibitors was investigated using plasmin derived from human plasma $\geq 2$ units/mg protein (Sigma, Munich, Germany). As the clinically relevant concentration of plasmin in $\mathrm{SMH}$ treated with VEGF-antagonists and rtPA is uncertain, two concentrations of plasmin were used: $5 \mu \mathrm{L}(200 \mu \mathrm{g})$ aflibercept and $5 \mu \mathrm{L}(50 \mu \mathrm{g})$ ranibizumab were incubated with $0.2 \mathrm{U}$ or $2 \mathrm{U}$ plasmin for $2 \mathrm{~h}$ at $37^{\circ} \mathrm{C}$ at a final volume of $10 \mu \mathrm{L}$. The mixtures were collected and separated in SDS-PAGE. In addition, for direct densitometric comparison of all VEGF inhibitors, including bevacizumab, we compared the effect of plasmin on equal amounts of the VEGF inhibitors $(50 \mu \mathrm{g})$, incubating them with $2 \mathrm{U}$ plasmin as described above.

\section{SDS-PAGE}

A 1:10 dilution of the samples in SDS-sample buffer (Novagen, Darmstadt, Germany) was separated in gel electrophoresis as described previously. ${ }^{15}$ As molecular weight control, Roti-Mark Prestained (Roth, Karlsruhe, Germany) or Precision Plus Protein Kaleidoscope (BioRad, München, Germany) protein standard were used. The pattern of protein bands was investigated in two different staining methods.

\section{Coomassie staining}

Coomassie staining was conducted as previously described. ${ }^{15}$

\section{Silver staining}

Silver staining was conducted using the Pierce Silver Stain Kit (Thermo Scientific, Bonn, Germany) as previously described. ${ }^{15}$

\section{Band detection}

All gels were photographed, using a Bio-Image System (ChemoBis, Biostep, Jahnsdorf, Germany) and dried in a standard procedure, using a 2\% glycerine solution and cellophane (Roth, Karlsruhe, Germany). Molecular weight and the density of bands were determined using TotalLab Software (Nonlinear Dynamics, Durham, North Carolina, USA). The range of the molecular weight of the corresponding bands was determined and the mean of this range is represented in the graphs (x-axis), plotted against the mean of the density (y-axis), representing mean and SD.

\section{Anti-VEGF efficacy of aflibercept and ranibizumab}

In order to determine the anti-VEGF efficacy of aflibercept or ranibizumab in the presence of rtPA, plasmin or $\mathrm{rtPA}$ and clotted human blood, the ability to inhibit VEGF detection by ELISA was quantified as previously described. ${ }^{18}$

Aflibercept and rtPA as well as ranibizumab and rtPA were incubated as described above. As controls, rtPA $(2 \mu \mathrm{g}$ in $10 \mu \mathrm{L}$ solution, plus $10 \mu \mathrm{L} \mathrm{NaCl})$, aflibercept $(400 \mu \mathrm{g}$ in $10 \mu \mathrm{L}$ solution, plus $10 \mu \mathrm{l} \mathrm{NaCl})$ and ranibizumab $(100 \mu \mathrm{g}$ in $10 \mu \mathrm{L}$ solution, plus $10 \mu \mathrm{L} \mathrm{NaCl}$ ) were separately incubated for $2 \mathrm{~h}$ at $37^{\circ} \mathrm{C}$. Aflibercept $(400 \mu \mathrm{g}$ and $200 \mathrm{ng})$ or ranibizumab $(100 \mu \mathrm{g})$, respectively, was incubated with $4 \mathrm{U}$ plasmin, to a final volume of $20 \mu \mathrm{L}$ for $2 \mathrm{~h}$ at $37^{\circ} \mathrm{C}$. As controls, plasmin ( $4 \mathrm{U}$ in $10 \mu \mathrm{L}$, plus $10 \mu \mathrm{g} \mathrm{NaCl})$, aflibercept $(400 \mu \mathrm{g}$ in $10 \mu \mathrm{g}$, plus $10 \mu \mathrm{g} \mathrm{NaCl})$ and ranibizumab $(100 \mu \mathrm{g}$ in $10 \mu \mathrm{g}$, plus $10 \mu \mathrm{g} \mathrm{NaCl})$, were separately incubated for $2 \mathrm{~h}$ at $37^{\circ} \mathrm{C}$. All mixtures were kept on ice until further use.

Intraoperative conditions were simulated to quantify the anti-VEGF efficacy of aflibercept and ranibizumab in the presence of rtPA and human blood. Whole blood was obtained from a healthy volunteer $(\mathrm{JH})$ as previously described. ${ }^{15} \mathrm{rtPA}$ and aflibercept as well as rtPA and ranibizumab were added at volumes and concentrations used in surgery (rtPA $50 \mu \mathrm{L} / 10 \mu \mathrm{g}$; aflibercept $(50 \mu \mathrm{L} / 2 \mathrm{mg})$ and ranibizumab $(50 \mu \mathrm{L} / 0.5 \mathrm{mg})$. The mixture was incubated for $30 \mathrm{~min}$ at $37^{\circ} \mathrm{C}$, centrifuged at $5600 \mathrm{rpm}$ for $10 \mathrm{~min}$ and the supernatant (approximately $500 \mu \mathrm{L}$ ) was collected and kept on ice until further use. As controls, rtPA, aflibercept and ranibizumab were added to the conditioned medium as described above.

Medium of confluent primary porcine RPE cells, second passage, was collected for $4 \mathrm{~h}$ as primary porcine RPE cells have been shown to secrete a significant amount of VEGF. ${ }^{18}$ To a volume of $500 \mu \mathrm{L}$ of conditioned medium, $20 \mu \mathrm{L}$ of the mixtures described above was added, incubated for $15 \mathrm{~min}$ at $37^{\circ} \mathrm{C}$ and centrifuged at $13000 \mathrm{rpm}$ for $5 \mathrm{~min}$. The supernatant was collected and a VEGF ELISA (Quantikine, R\&D Systems, Wiesbaden, Germany) was performed as previously described. ${ }^{18}$

\section{RESULTS}

\section{Interaction studies}

\section{Recombinant tissue plasminogen activator}

We investigated the effect of rtPA on the band pattern of aflibercept and ranibizumab when applied in equal volumes, assessed in two different protein stains, and evaluating the molecular weight of the bands. The band pattern of aflibercept and ranibizumab did not change in rtPA coincubation in Coomassie staining (figure 1A). The molecular weight of the bands displayed for aflibercept (56 kDa (main band), $43 \mathrm{kDa}, 37 \mathrm{kDa}, 31 \mathrm{kDa}, 26 \mathrm{kDa}, 23 \mathrm{kDa}$ ) and ranibizumab $(24 \mathrm{kDa})$ showed not difference between treated or untreated antagonists. The band appearing at $62 \mathrm{kDa}$ in treated ranibizumab corresponds to rtPA. Also, the distribution of the bands did not change. The reduction of the band intensity is due to a dilution effect of the mixture applied to the gel. In silver staining, we also did not find any differences between untreated and rtPA treated aflibercept (68 kDa (main band), $41 \mathrm{kDa}, 36 \mathrm{kDa}$, 
rtPA

A Coomassie

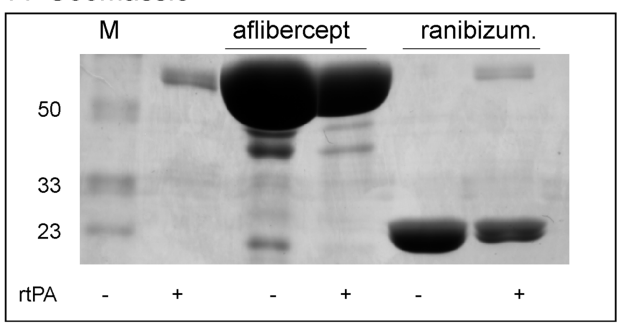

B Silver

\begin{tabular}{|c|c|c|c|}
\hline \multirow[b]{2}{*}{50} & M & aflibercept & ranibizum. \\
\hline & & & \\
\hline 33 & & 5 & \\
\hline 23 & & 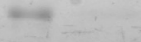 & \\
\hline rtPA & - & + & + \\
\hline
\end{tabular}

\section{Plasmin}

\section{Coomassie}

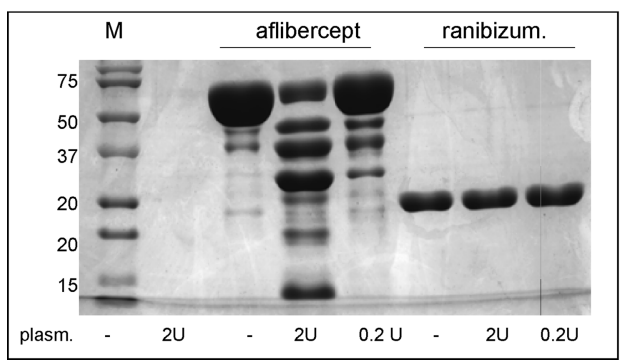

D Silver

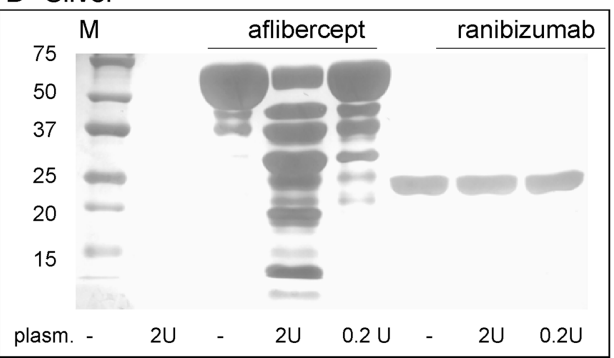

Figure 1 Compatibility of ranibizumab and aflibercept with recombinant tissue plasminogen activator (rtPA) and plasmin. Equal volumes of aflibercept $(200 \mu \mathrm{g})$ and ranibizumab $(50 \mu \mathrm{g})$ were incubated with equal volumes of rtPA (A and B) or with plasmin $(0.2 \mathrm{U}$ and $2 \mathrm{U})(\mathrm{C}$ and $\mathrm{D})$ for $2 \mathrm{~h}$ at $37^{\circ}$, separated on $12 \%$ SDS-PAGE and stained in Coomassie blue (A and C) or silver (B and D). (A) Incubation with rtPA, stained with Coomassie blue, (B) Incubation with rtPA, stained with silver, (C) Incubation with plasmin, stained with Coomassie blue, (D) Incubation with plasmin, stained with silver. No differences between treated and untreated inhibitors were found for either substance. Representative gels are shown ranibizum., ranibizumab; plasm, plasmin.

A Coomassie staining, $50 \mu \mathrm{g}$ VEGF antagonist

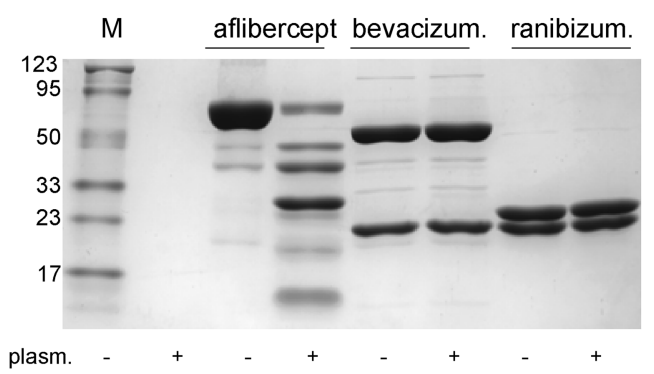

C Bevacizumab, densitrometric evaluation

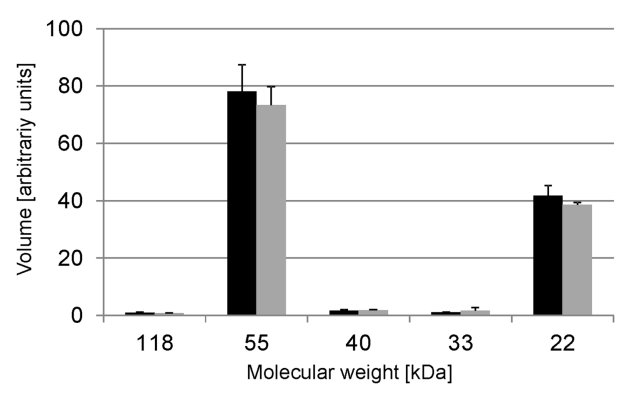

B Aflibercept, densitrometric evaluation

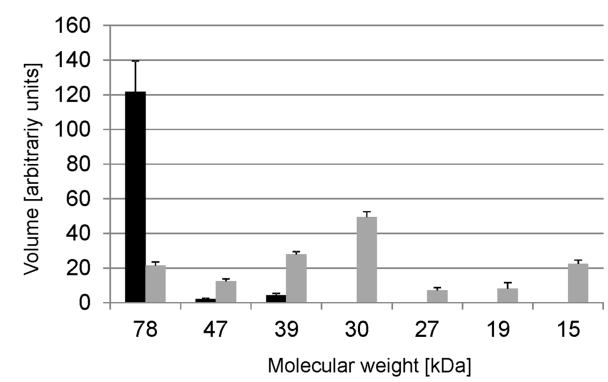

D Ranibizumab, densitrometric evaluation

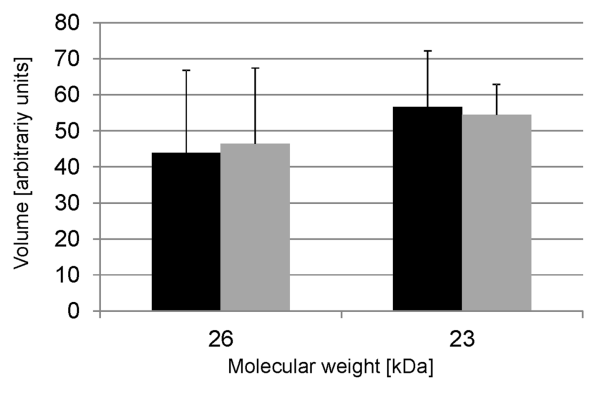

with plasmin

Figure 2 Densitometric evaluation of plasmin-incubated inhibitors, Coomassie blue. $50 \mu \mathrm{g}$ of aflibercept, ranibizumab or bevacizumab were incubated with $2 \mathrm{U}$ plasmin for $2 \mathrm{~h}$ at $37^{\circ}$, separated on $12 \%$ SDS-PAGE and stained in Coomassie blue. (A) Representative gel. (B) Densitometric evaluation of protein bands found for aflibercept with and without $2 \mathrm{U}$ plasmin. (C) Densitometric evaluation of protein bands found for bevacizumab with and without $2 \mathrm{U}$ plasmin. (D) Densitometric evaluation of protein bands found for ranibizumab with and without $2 \mathrm{U}$ plasmin. Plasmin in either activity had no effect on the protein bands of ranibizumab or bevacizumab. However, the band distribution and intensity changed for aflibercept treated with $2 \mathrm{U}$ plasmin. Bars depict mean density and SD, plotted against molecular weight of the respective band. Ranibizum., ranibizumab, bevacizum., bevacizumab, w/o, without. 
$31 \mathrm{kDa}, 25 \mathrm{kDa}, 20 \mathrm{kDa})$ or ranibizumab $(24 \mathrm{kDa})$, neither in molecular weight nor in distribution of the bands (figure 1B).

\section{Plasmin}

Plasmin is the main product of rtPA activity. We investigated the effect of plasmin on molecular weight and distribution of aflibercept $(200 \mu \mathrm{g})$ and ranibizumab $(50 \mu \mathrm{g})$. When applying either $2 \mathrm{U}$ or $0.2 \mathrm{U}$ of plasmin, the band pattern of ranibizumab in Coomassie did not change $(25 \mathrm{kDa})$ (figure $1 \mathrm{C})$. In contrast, the band pattern and distribution changed for aflibercept when incubated with $2 \mathrm{U}$ (untreated $60 \mathrm{kDa}$ (main band), $46 \mathrm{kDa}$, $30 \mathrm{kDa}, 25 \mathrm{kDa}$; plasmin additional bands: $23 \mathrm{kDa}, 20 \mathrm{kDa}$, $11 \mathrm{kDa}$ ) or, to a lesser degree, at $0.2 \mathrm{U}$ plasmin (additional band at $23 \mathrm{kDa}$ ) as seen in Coomassie staining (figure 1C). Silver staining confirmed these results, with no change in molecular weight or distribution for ranibizumab $(24 \mathrm{kDa}$ ) (figure 1D), but changes found in molecular weight and distribution for aflibercept when combined with plasmin ( $2 \mathrm{U}$ additional bands $21 \mathrm{kDa}, 19 \mathrm{kDa}, 15 \mathrm{kDa}$ and $13 \mathrm{kDa} ; 0.2 \mathrm{U}$ additional bands $21 \mathrm{kDa}$, and $19 \mathrm{kDa}$ ) (figure 1D).

In order to allow a direct densitometric comparison of band obtained under plasmin incubation, we repeated the experiments applying $50 \mu \mathrm{g}$ of the respective VEGF-antagonist and incubated them with $2 \mathrm{U}$ plasmin. To directly compare with aflibercept and ranibizumab, we also included bevacizumab in our study. In this approach, the band pattern of ranibizumab did not change either in Coomassie (figure 2D) or silver staining (figure 3D). Similarly, the pattern of bevacizumab did not change, in either staining (figure 2C and $3 \mathrm{C}$ ). In contrast, aflibercept displays a clear change of band pattern with additional low molecular bands and a clear decrease of the signal of the original bands, both shown in Coomassie (figure 2B) and silver staining (figure $3 \mathrm{~B}$ ).

\section{Anti-VEGF efficacy of aflibercept and ranibizumab}

We have investigated the efficacy of the respective VEGF-antagonist assessing the ability to inhibit VEGF detection in RPE supernatant in ELISA. As expected, aflibercept $(400 \mu \mathrm{g}$ in $500 \mu \mathrm{L}$ supernatant) and ranibizumab $(100 \mu \mathrm{g}$ in $500 \mu \mathrm{L}$ supernatant) virtually completely inhibited VEGF detection at the given concentrations (figure 4). ${ }^{19}$ When coincubated with rtPA $(2 \mu \mathrm{g})$, both antagonists still completely inhibited VEGF detection (figure 4A). We also investigated the ability of ranibizumab and aflibercept, respectively, to inhibit VEGF when coincubated with rtPA and blood in volumes and concentrations used in surgery with $10 \mu \mathrm{grtPA}, 2 \mathrm{mg}$ aflibercept and $0.5 \mathrm{mg}$ ranibizumab, and found no reduction of efficacy (figure 4C). ${ }^{89}$

When incubated with $2 \mathrm{U}$ plasmin, ranibizumab $(100 \mu \mathrm{g}$ in $500 \mu \mathrm{L}$ supernatant) still completely inhibited VEGF detection. Despite of its cleavage, aflibercept $(400 \mu \mathrm{g}$ in $500 \mu \mathrm{L}$ supernatant) completely inhibited VEGF when coincubated with plasmin (figure 4B). However, when using lower concentrations of aflibercept (200 ng in $500 \mu \mathrm{L}$ supernatant) at borderline efficacy, ${ }^{19}$ the incubation with plasmin completely abolished the efficacy of aflibercept to inhibit VEGF (figure 4D).

\section{DISCUSSION}

The treatment of neovascular AMD with SMH with a combined application of rtPA and anti-VEGF is a promising novel therapeutic approach which is currently introduced into clinical

B Aflibercept, densitrometric evaluation
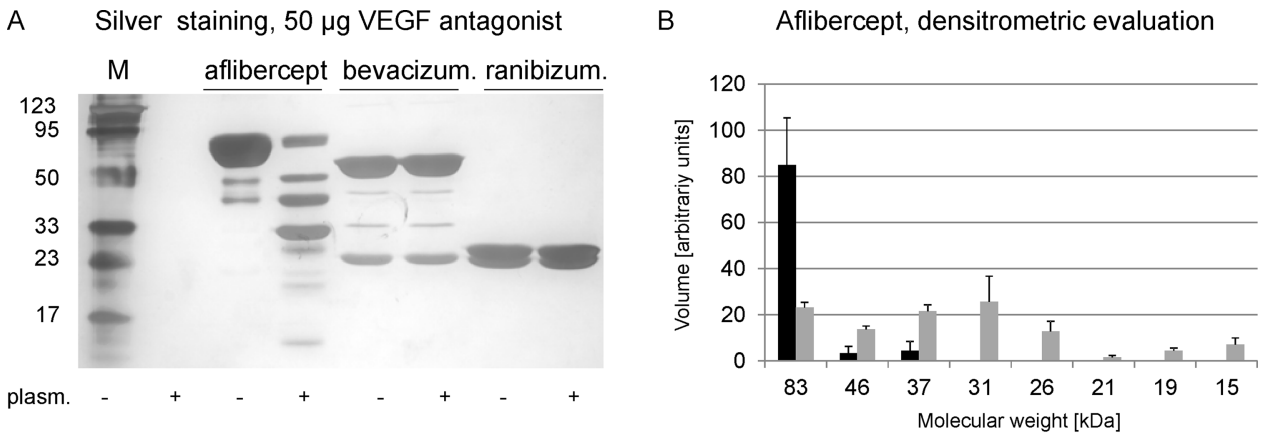

D Ranibizumab, densitrometric evaluation
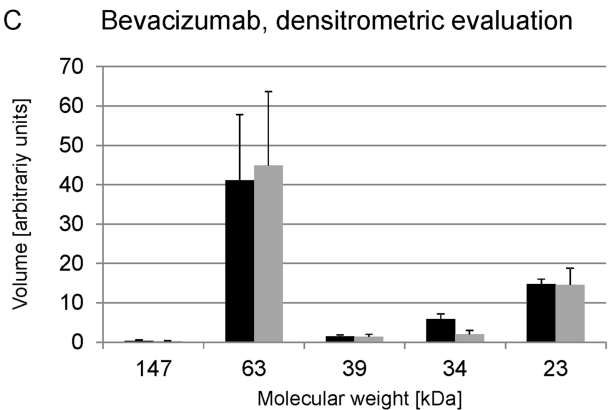

w/o plasmin

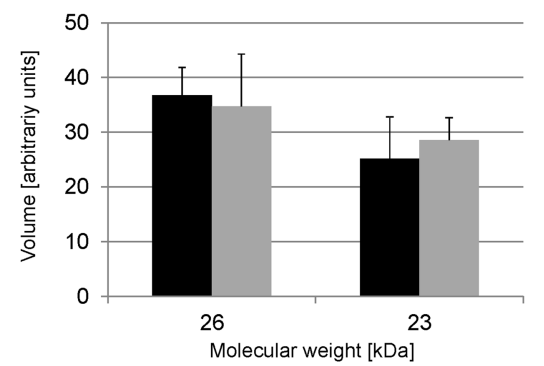

with plasmin

Figure 3 Densitometric evaluation of plasmin-incubated inhibitors, silver staining. $50 \mu \mathrm{g}$ of aflibercept, ranibizumab or bevacizumab were incubated with $2 \mathrm{U}$ plasmin for $2 \mathrm{~h}$ at $37^{\circ}$, separated on $12 \%$ SDS-PAGE and stained in silver staining. (A) Representative gel. (B) Densitometric evaluation of protein bands found for aflibercept with and without $2 \mathrm{U}$ plasmin. (C) Densitometric evaluation of protein bands found for bevacizumab with and without $2 \mathrm{U}$ plasmin. (D) Densitometric evaluation of protein bands found for ranibizumab with and without $2 \mathrm{U}$ plasmin. Plasmin in either activity had no effect on the protein bands of ranibizumab or bevacizumab. However, the band distribution and intensity changed for aflibercept treated with $2 \mathrm{U}$ plasmin. Bars depict mean density and SD, plotted against molecular weight of the respective band. Ranibizum., ranibizumab, bevacizum., bevacizumab, w/o, without. 
A

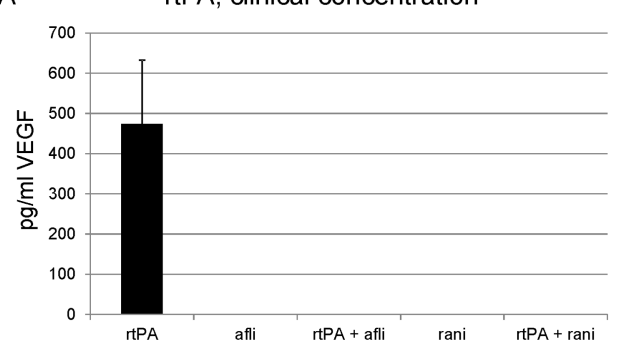

C

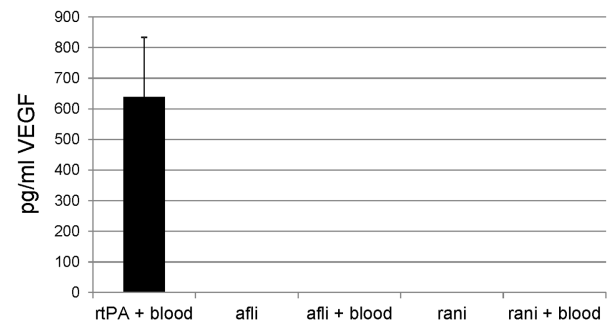

B

plasmin, clinical concentration

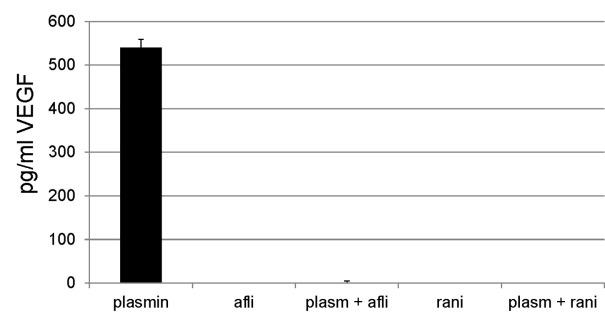

$\mathrm{D}$

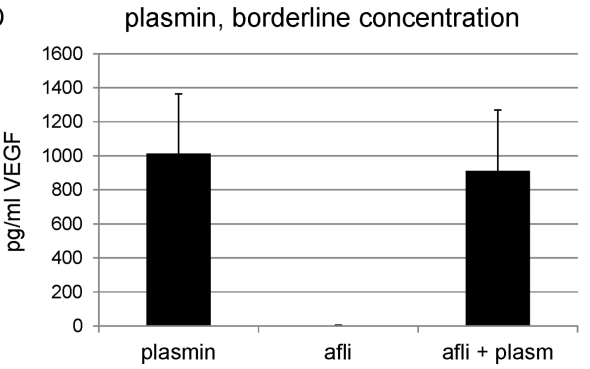

Figure 4 Antivascular endothelial growth factor (anti-VEGF) efficacy of aflibercept and ranibizumab. Anti-VEGF efficacy was investigated in ELISA. (A) Aflibercept $(400 \mu \mathrm{g})$ and ranibizumab $(100 \mu \mathrm{g})$ in equal volume were incubated with recombinant tissue plasminogen activator (rtPA) $(2 \mu \mathrm{g})$ for $2 \mathrm{~h}$ at $37^{\circ}$ and the mixture was added to retinal pigment epithelium (RPE) cell culture supernatant. As control, aflibercept and ranibizumab, respectively, were incubated without rtPA and applied to RPE supernatant, both inhibitors, with or without rtPA, completely abolished VEGF detection. (B) Aflibercept $(400 \mu \mathrm{g})$ and ranibizumab $(100 \mu \mathrm{g})$ in equal volume were incubated with plasmin $(4 \mathrm{U})$ for $2 \mathrm{~h}$ at $37^{\circ}$ and the mixture was added to RPE cell culture supernatant. As control, aflibercept and ranibizumab, respectively, were incubated without plasmin and applied to RPE supernatant. Both inhibitors, with or without rtPA, completely abolished VEGF detection. (C) A human blood clot was incubated with $10 \mu \mathrm{g}$ rtPA and $2 \mathrm{mg}$ aflibercept or $0.5 \mathrm{mg}$ ranibizumab for $30 \mathrm{~min}$. The supernatant was collected and added to RPE medium. Both inhibitors, with or without incubation with blood and rtPA, completely abolished VEGF detection. (D) Aflibercept at a concentration at the border of its efficacy $(200 \mathrm{ng})$ was incubated with plasmin as described above. At this concentration, after incubation with plasmin, aflibercept did not inhibit VEGF detection any longer. afli, aflibercept, plasm, plasmin, rani, ranibizumab.

practice. Here, we investigated the suitability of ranibizumab and aflibercept for subretinal coapplication with rtPA in patients.

We simulated the surgical situation in an established in vitro model. ${ }^{15}$ Accordingly, in order to cover the range of likely mixing ratios, we have used in our experiments equal volumes of the respective inhibitor and rtPA, and two different activities of plasmin. In order to exclude different concentrations of the respective VEGF inhibitor as a possible cause of significant cleavage when incubated together with plasmin and to allow a reliable densitometric evaluation of the electrophoresis bands, we repeated the experiments with plasmin with equal concentrations of the VEGF inhibitors. To directly compare these results with the previously published data on bevacizumab, ${ }^{15}$ we included bevacizumab in this experiment (figures 2 and 3 ).

The present findings clearly indicate that ranibizumab is not compromised when coapplied with rtPA, or with plasmin, which is the main product of enzymatic rtPA activity. As seen with both staining agents, the electrophoresis band patterns clearly show no additional bands when ranibizumab is coincubated with either rtPA or plasmin, hence ranibizumab remains structurally intact (figures 1-3). Neither of the substances cleaves ranibizumab. Moreover, the ELISA experiments clearly show that neither rtPA, plasmin nor proteases activated during fibrinolysis of a blood clot functionally inactivate ranibizumab, indicating that the antiangiogenic efficacy of ranibizumab remains intact in the presence of rtPA with or without human blood or in the presence of plasmin.

By contrast, while aflibercept remained structurally intact when coincubated with rtPA, both stains clearly showed that aflibercept is cleaved by plasmin (figures 2 and 3). Furthermore, when aflibercept was not applied in excess amounts but in concentration at the borderline of its efficacy, ${ }^{19}$ its ability to inhibit VEGF was clearly reduced by plasmin, indicating reduced antiangiogenic efficacy (figure 4).

The densitometric evaluation of bevacizumab when coapplied with plasmin confirmed our previously published data, showing that bevacizumab is not compromised by plasmin. ${ }^{15}$

The present findings may be clinically relevant because our in vitro experiments suggest that aflibercept, in contrast with ranibizumab or bevacizumab may be partially inactivated by plasmin when coapplied with rtPA in the subretinal space. However, the question remains whether these results indicate an impaired performance of aflibercept when clinically used in combination with rtPA. While the cleavage is obvious, the effect on VEGF inhibition is only seen when aflibercept is present at borderline concentrations. Hence, even though plasmin may cleave aflibercept, VEGF inhibition is likely to still occur. However, following intraocular application, VEGF antagonists are cleared from the eye with an approximate half-life of $3-9$ days, ${ }^{20}$ and so the cleavage of aflibercept may reduce the time frame in which it can exert its antiangiogenic function.

In conclusion, we demonstrate the absence of cleavage of ranibizumab by rtPA or plasmin and the absence of a functional inactivation of ranibizumab by rtPA with or without whole blood or by plasmin. In clinical practice, rtPA and ranibizumab can be coapplied to simultaneously clear SMH and reduce choroidal new vessel activity. Furthermore, we demonstrate that plasmin but not rtPA cleaves aflibercept. The anti-VEGF efficacy of aflibercept may be compromised when not present in excess amounts. 
Contributors AK, SG, FT, JR, JH: study design, data interpretation, manuscript review and approval; AK, SG: data acquisition; AK, JH: manuscript preparation.

Competing interests Independent of this study, AK has been a consultant for and received research grants and lecture fees from Novartis Pharma. Independent of this study, JH has received lecture fees and travel grants from Novartis Pharma.

Ethics approval Porcine eyes were obtained with permission from the local abattoir, where the animals are killed for the purpose of food production and the eyes are regularly removed from the slaughtered animals due to legal regulations (Tier-LMHV (Anlage 5 zu §7 Satz 2, Kapitel III, Nr. 2.4). The usage of the eyes for experimental purposes was conducted in agreement with the animal welfare officer of the University of Kiel.

Provenance and peer review Not commissioned; externally peer reviewed.

\section{REFERENCES}

1 Avery RL, Fekrat S, Hawkins BS, et al. Natural history of subfoveal hemorrhage in age-related macular degeneration. Retina 1996;16:183-9.

2 Glatt H, Machemer R. Experimental subretinal hemorrhage in rabbits. Am J Ophthalmol 1982;94:762-73.

3 Toth CA, Mourse LS, Hjemeland LM, et al. Fibrin early retinal damage after experimental subretinal hemorrahge. Arch Ophthalmol 1991;109:723-9.

4 Bhisitkul RB, Winn BJ, Lee OT, et al. Neuroprotective effect of intravitreal triamcinolone acetonide against photoreceptor apoptosis in a rabbit model of subretinal hemorrhage. Invest Ophthalmol Vis Sci 2008;49:4071-7.

5 El Baba F, Jarrett WH. Massive hemorrhage complicating age-related macular degeneration: Clinicopathologic correlation and role of anticoagulants. Ophthalmology 1986;93:1581-92.

6 Ip MS, Scott IU, Brown GC, et al. Anti-vascular endothelial growth factor pharmacotherapy for age-related macular degeneration: a report by the American Academy of Ophthalmology. Ophthalmology 2008;115:1837-46.

7 Schouten JS, La Heij EC, Webers CA, et al. A systematic review on the effect of bevacizumab in exudative age-related macular degeneration. Graefes Arch Clin Exp Ophthalmol 2009;247:1-11.

8 Treumer F, Klatt C, Roider J, et al. Subretinal co-application of recombinant tissue plasminogen activator and Bevacizumab for neovascular age-related macular degeneration with submacular hemorrhage. Br J Ophthalmol 2010;94:48-53.
9 Treumer F, Roider J, Hillenkamp J. Long-term outcome of subretinal co-application of rtPA and bevacizumab followed by repeated intravitreal anti-VEGF injections for neovascular AMD with submacular hemorrhage. Br J Ophthalmol 2012;96:708-13.

10 Meyer $\mathrm{CH}$, Scholl HP, Eter $\mathrm{N}$, et al. Combined treatment of acute subretinal haemorrhages with intravitreal recombined tissue plasminogen activator, expansile gas and bevacizumab: a retrospective pilot study. Acta Ophthalmol 2008;86:490-4.

11 Guthoff R, Guthoff T, Meigen T, et al. Intravitreous injection of bevacizumab, tissue plasminogen activator, and gas in the treatment of submacular hemorrhage in age-related macular degeneration. Retina 2011;31:36-40.

12 Haupert CL, McCuen BW II, Jaffe GJ. Pars plana vitrectomy, subretinal injection of tissue plasminogen activator, and fluid-gas exchange for displacement of thick submacular hemorrhage in age-related macular degeneration. Am J Ophthalmol 2001;131:208-15.

13 Olivier S, Chow DR, Packo KH. Subretinal recombinant tissue plasminogen activator injection and pneumatic displacement of thick submacular hemorrhage in age-related macular degeneration. Ophthalmology 2004;111:1201-8.

14 Hillenkamp J, Surguch V, Framme C, et al. Management of submacular hemorrhage with intravitreal versus subretinal injection of recombinant tissue plasminogen activator. Graefes Arch Clin Exp Ophthalmol 2010;248:5-11.

15 Klettner A, Puls S, Treumer F, et al. Compatibility of recombinant tissue plasminogen activator (rtPA) and bevacizumab co-applied for neovascular age-related macular degeneration with submacular hemorrhage. Arch Ophthalmol 2012;130:875-81.

16 Mars WM, Zarnegar R, Michalopoulos GK. Activation of hepatocyte growth factor by the plasminogen activators UPA and tPA. Am J Pathol 1993;143:949-858.

17 Su EJ, Fredriksson L, Geyer M, et al. Activation of PDGF-CC by tissue plasminogen activator impairs blood-brain barrier integrity during ischemic stroke. Nat Med 2008:14:731-7.

18 Klettner A, Roider J. Comparison of bevacizumab ranibizumab, and pegaptanib in vitro: efficiency and possible additional pathways. Invest Ophthalmol Vis SCi 2008;49:4523-7

19 Klettner A, Recber M, Roider J. Comparison of the efficacy of aflibercept, ranibizumab and bevaiczumab in RPE/choroid organ culture. Graefes Arch Clin Exp Ophthalmol 2014;252:1593-8

20 Klettner A. VEGF-A and its inhibitors in age-related macular degenerationpharmacokinetic differences and their retinal and systemic implications. J Biochem Pharmacol Res 2014;2:8-20. 